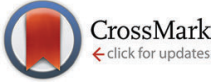

Cite this: J. Mater. Chem. C, 2016, 4,6302

\section{Transition dipole moment orientation in films of solution processed fluorescent oligomers: investigating the influence of molecular anisotropy $\dagger$}

\author{
Alessia Senes, ${ }^{\text {ab }}$ Stefan C. J. Meskers, ${ }^{b}$ Wijnand M. Dijkstra, ${ }^{b}$ Jacobus J. van Franeker, ${ }^{b c}$ \\ Stéphane Altazin, ${ }^{d}$ Joanne S. Wilson ${ }^{a}$ and René. A. J. Janssen ${ }^{\star b}$
}

\begin{abstract}
The low light-outcoupling efficiency of organic light emitting diodes (OLEDs) is limiting their performance. Orientation of the transition dipole moment of the emitting molecules in the plane of the diodes can improve the luminance of OLEDs. While the orientation of evaporated small-molecule materials has been studied in the past few years, not much is known about solution processed small molecules and short oligomers, and it is not clear yet which parameters influence their orientation in the film. In this work we study a series of short conjugated $p$-phenylene vinylene oligomers (OPVn), consisting of an increasing number of repeating phenyl rings ( $n$ from 2 to 7), which are introduced into a small-molecule host matrix. By measuring the angular distribution of $p$-polarised fluorescence intensity from thin solution processed films, we determine the average orientation of the transition dipole moment of the emitters in the host matrix. We find that for longer oligomers $(n=6,7)$, the transition dipole moments align more horizontally, with ratios of horizontally to vertically oriented dipoles up to $80: 20$. The preferential horizontal alignment is related to the aggregation of the emitter molecules.
\end{abstract}

Received 24th October 2015, Accepted 2nd June 2016

DOI: $10.1039 / \mathrm{c} 5 \mathrm{tc0} 03481 \mathrm{~g}$

www.rsc.org/MaterialsC outcoupling efficiency is usually around $20 \%$, drastically limiting the external quantum efficiency (EQE) of the devices. This is due to various losses inside OLEDs, such as waveguiding of the light in the substrate and OLED layers, and cathode quenching. ${ }^{2}$ Various light extraction techniques have proven to be useful in improving the outcoupling efficiency in OLEDs such as external outcoupling foils, internal light extraction layers with a high refractive index and corrugated cathodes. ${ }^{2}$ Recently the importance of the orientation of the transition dipole moment of the emitters in small-molecule materials has become apparent. By changing the orientation of the transition dipole moments of the emitting molecule from random to predominantly horizontal, in the plane direction, the light outcoupling efficiency can be improved. ${ }^{3-5}$ Orientation of the transition dipole moment in the plane of the substrate can increase the light outcoupling efficiency of the OLED by 1.5 times compared to devices with randomly oriented emitters. ${ }^{6}$ Moreover, changing the molecular orientation can also improve the charge carrier transport in the charge transport layers. ${ }^{7}$

Most studies on molecular orientation have focused on alignment in vapour deposited films of small-molecule materials. ${ }^{8}$ One of the factors influencing the orientation is the anisotropy in the molecular shape. It has been observed that molecules with a long, rod-like shape, planar oblate shape or long and winding shape tend to show optical anisotropy in the film. ${ }^{9,10}$ On the other hand molecules with a more globular shape have optical 
properties that are almost isotropic. ${ }^{8}$ Another factor that is reported to influence the orientation of the molecules is the formation of $\mathrm{C}-\mathrm{H} \cdots \mathrm{N}$ intermolecular hydrogen bonds. ${ }^{11}$ The hydrogen bond formation can promote the in-plane alignment of the transition dipole moments.

Also the presence of permanent electrical dipole moments in molecules may influence the orientation of molecules in vapour deposited films. A correlation was found between the permanent dipole moment and the preferential horizontal orientation of the transition dipole moment in a study of seven different iridium complexes. ${ }^{12}$ Emitters with a smaller permanent dipole moment were found to show a higher degree of horizontal orientation of the transition dipole moment. This correlation likely only holds for a series of molecules with a fixed mutual orientation of the transition dipole moment and the permanent dipole moment. In a recent study an alternative mechanism was proposed for iridium dyes in which the inherent asymmetry at the surface of the growing film, created by the acetylacetonate ligand of the iridium dye, promotes the alignment of the dopant. ${ }^{13}$

So far studies on the orientation of transition the dipole moment in small-molecule materials for OLED applications have focused mainly on evaporated materials. For luminescent conjugated polymers preferential horizontal orientation in solution processed layers is known. ${ }^{14-17}$ High degrees of horizontal orientation have also been reported for a light-emitting heptafluorene oligomer in a solution processed host/guest system. ${ }^{18}$ The preferential orientation of small emitter molecules for OLEDs has been studied by Yokoyama et al. For a number of small molecules they found that the degree of horizontal molecular orientation of small-molecule spin-coated films is inherently lower than that of the corresponding vacuum evaporated films. ${ }^{19,20}$

In this study we investigate whether the horizontal alignment of light emitting oligomeric guests in a small molecule host can be induced in layers deposited from solution by varying the aspect ratio of the oligomers. We studied a series of $\pi$-conjugated methyl end-capped $p$-phenylene vinylene oligomers (OPVn), in which the number of phenyl rings, $n$, varies from 2 to 7 (Fig. 1). The host matrix we use is $2,2^{\prime}, 2^{\prime \prime}$-benzene-1,3,5-triyltris(1-phenyl$1 H$-benzimidazole) (TPBi). In evaporated layers, TPBi molecules have been shown to be randomly oriented on the substrate. ${ }^{8}$
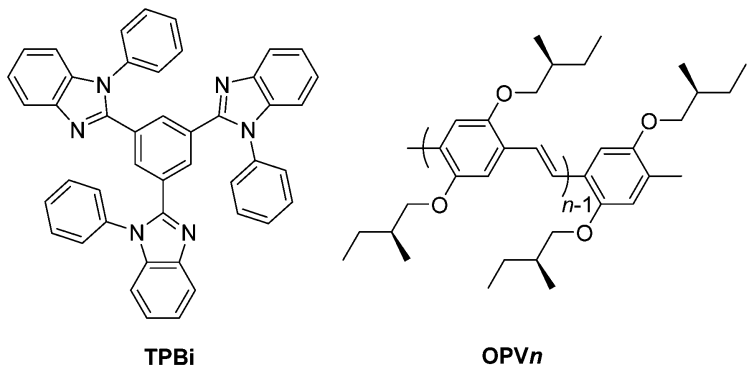

Fig. 1 Chemical structure of host and guest molecules used in this work (a) Host: TPBi. (b) Guest: OPV $n$ with $n$ being the number of phenyl rings ranging from 2 to 7 .

\section{Experimental}

\section{Materials}

The synthesis of the OPVn molecules has been described previously. ${ }^{21}$ TPBi was obtained from Lumtec. The two components of the host/guest system were mixed in a 9:1 weight ratio and dissolved in chloroform, $1 \mathrm{w} / \mathrm{v} \%$. For OPV4, OPV6 and OPV7 also films with a host/guest weight ratio of 49:1 were prepared. The mixed solution of TPBi and OPVn was stirred at $40{ }^{\circ} \mathrm{C}$ overnight and cooled down before use. Solutions were filtered through a $0.22 \mu \mathrm{m}$ PTFE filter and deposited by spin coating at a speed of $1200 \mathrm{rpm}$ on quartz substrates. The layer thickness was around $40 \mathrm{~nm}$ for all layers, as measured by surface profilometry.

\section{Angular dependent fluorescence}

In order to analyse the orientation of the transition dipole moment of the emitters in solution processed layers, we measured the angular dependence of the $p$-polarised fluorescence intensity. The setup used (Fig. 2) was previously described by Brütting et $a l^{22}$ The layers are excited with the focused light of a $365 \mathrm{~nm}$ LED, and the fluorescent light emitted through the quartz substrate, which is optically coupled to a quartz hemicylinder through matched refractive index oil, is $p$-polarised using a polarizer and collected using a spectrometer. The fluorescence is measured at different angles, from $0^{\circ}$ to $80^{\circ}$, in steps of $2^{\circ}$. The measurements were performed in an inert $\mathrm{N}_{2}$ atmosphere $\left(\mathrm{H}_{2} \mathrm{O}\right.$ and $\left.\mathrm{O}_{2}<1 \mathrm{ppm}\right)$. The measurements were repeated on different samples to ensure reproducible and consistent results.

The optical constants (refractive index, $n$, and extinction coefficient $k$ ) of the pure host material (Fig. S1, ESI $\dagger$ ) were determined experimentally by spectroscopic ellipsometry at various angles of incidence and transmission intensity measurements at $0^{\circ}$ (perpendicular incidence). The data were analysed using Woollam CompleteEASE software (version 4.92). Ellipsometry on layers of the pure host did not reveal any indication for optical anisotropy and so the optical properties of the host were taken to be the same in all directions.

Absorption spectra were obtained from transmission measurements on the host/guest films on quartz substrates. For clarity the

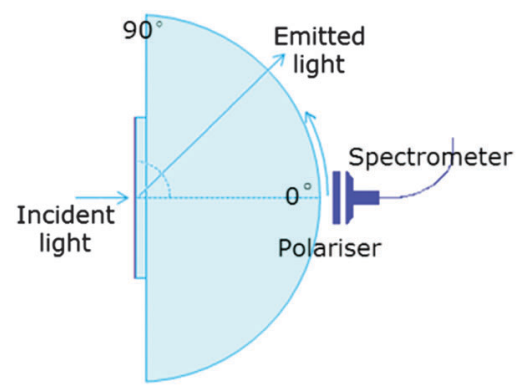

Fig. 2 Layout of the experimental setup used for the angle-dependent fluorescence measurement. The organic layer whose luminescence is measured is on top of a quartz substrate. The uncovered bottom of the quartz substrate is in optical contact with a quartz hemicylinder through a refractive index matching oil. The organic layer is excited using a UV LED and the fluorescence is collected using a polarizer and a fibre-coupled spectrometer. 
contribution of the host material to the absorption, peaking at $306 \mathrm{~nm}$ and a baseline were subtracted from the spectra.

The experimental angular dependencies of the $p$-polarised fluorescence intensities of the TPBi/OPV $n$ layers were combined with the optical constants of pure TPBi and used as input for optical simulations using Setfos 4.0 (Fluxim AG, Switzerland). Setfos 4.0 solves the Maxwell equations for the set of stacked layers, with oriented dipole oscillators embedded in the host layer as sources of radiation representing the emitting molecules. ${ }^{23}$ Both far field radiation and near field radiation patterns of the dipoles are simulated. In the modelling it is assumed that photoselection in the excitation of dipole emitters is absent and that every fluorescent molecule is excited with equal probability regardless of its orientation. This is a reasonable assumption because $365 \mathrm{~nm}$ excitation is at higher energy than the lowest energy, parallel polarized, $S_{0} \rightarrow S_{1}$ absorption band (except for OPV2) as can been in the optical absorption spectra of the OPVn molecules (Fig. S2, $\mathrm{ESI} \dagger)$. Furthermore, the high concentrations of 2 and $10 \mathrm{wt} \%$ stimulate energy transfer and randomization among the orientation of excited OPV $n$ molecules before they emit. By comparing to the experimental data the optical simulations allow quantifying the degree of orientation of the transition dipole moments.

\section{Microscopy}

The host/guest layers for transmission electron microscopy (TEM) were processed from chloroform solutions on top of a layer of poly(3,4-ethylenedioxythiophene):poly(styrene sulfonate) (Clevios PEDOT:PSS, Heraeus) on glass substrates. By immersing the layer stack in deionized water, the PEDOT:PSS layer is dissolved and the host/guest film floats on the surface. The host/guest layer is picked up onto 200 square mesh copper TEMgrids. TEM images were taken on a Tecnai G2 Sphera, operating at $200 \mathrm{kV}$, using a magnification of $5000 \times$ and a defocus of $-10 \mu \mathrm{m}$. Atomic force microscopy (AFM) was performed using a Veeco Dimension 3100 microscope in tapping mode with a scan rate of $1 \mathrm{~Hz}$ and ppp-NCHR-50 tips from Nanosensors, on the same layers as used in the fluorescence measurements.

\section{Results and discussion}

\section{Transition dipole moment orientation and layer analysis}

All TPBi/OPVn host/guest layers studied showed bright fluorescence originating from the OPVn molecules. In Fig. 3 we show the absorption and fluorescence spectra of the TPBi/OPV4 (10 wt\%) layer. The absorption with the maximum at $450 \mathrm{~nm}$ and fluorescence with the maximum at $550 \mathrm{~nm}$ correspond to the allowed optical transition between the ground state $\left(S_{0}\right)$ and the lowest singlet excited state $\left(S_{1}\right)$ of the OPV4 molecule. The transition dipole moment for the $\mathrm{S}_{0}-\mathrm{S}_{1}$ transition is known to be predominantly oriented along the long axis of the molecule, along the system series of $\pi$-conjugated bonds. ${ }^{24-26}$

Fig. 4 shows the angular dependence of the $p$-polarised fluorescence intensity of the TPBi/OPVn films. For each film, the intensity was recorded at the wavelength of maximum intensity and normalized to the intensity of the fluorescence

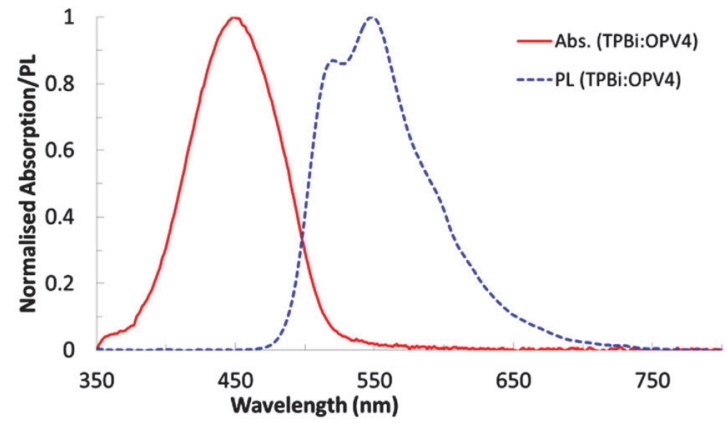

Fig. 3 Absorption (solid line) and fluorescence (dashed line) spectra of a TPBi/OPV4 (10 wt\%) layer.

in the direction of the surface normal of the film $\left(0^{\circ}\right)$. For the films containing short oligomers $(n \leq 5)$ we notice that the fluorescence intensity remains relatively constant for angles up to $\sim 40^{\circ}$ and then rapidly increases. The fluorescence intensity shows a narrow peak at $43^{\circ}$ and a broader maximum at around $53^{\circ}$. The high normalised intensities $(>1)$ at high angles indicate a preferential vertical orientation of the transition dipole moments. A transition dipole moment oriented along the normal of the films would radiate waves with the propagation direction predominantly in the plane of the film. The intensity emitted in the direction along the surface normal is minimal for a vertical dipole. In contrast, for the OPV7 film we find that the fluorescence intensity at $53^{\circ}$ is lower than at $0^{\circ}$, indicating a preferred orientation of the transition dipole moments in the plane of the film. The minimum in $p$-polarised fluorescence intensity at $42^{\circ}$ is related to the Brewster angle for internal reflection at the host/air interface where light can couple out effectively in the backward direction. Thus qualitative analysis of the intensity profiles shown in Fig. 4 indicates the preferential vertical orientation of the shorter oligomers and more horizontal orientation of the longer OPVn guest molecules in the solution processed films.

In order to quantify the orientation of the transition dipole moments we combine the measurements with optical simulation of the angular intensity profile of the $p$-polarized emission.

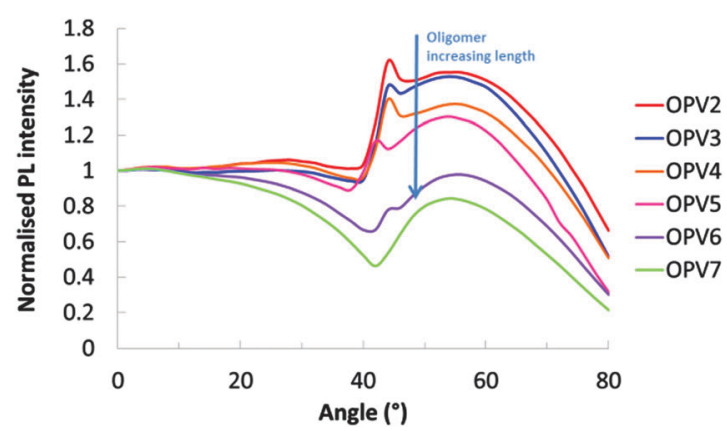

Fig. 4 Angular dependence of the $p$-polarised fluorescence of solution processed TPBi/OPVn (10 wt\%) layers. The fraction of horizontal dipoles increases upon increasing the length of the OPVn molecule. Layers containing OPV6 and OPV7 show preferential horizontal transition dipole moment orientation. 


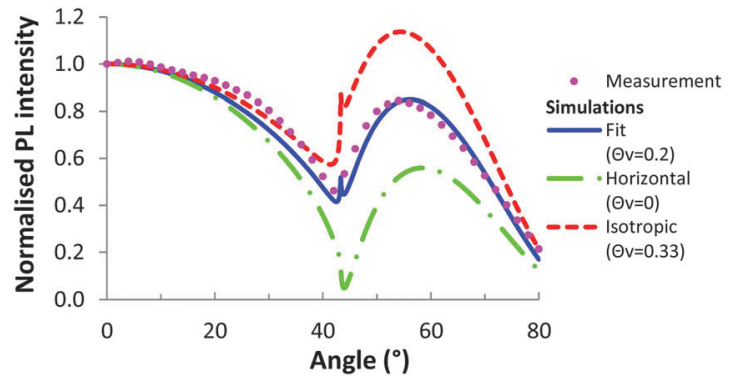

Fig. 5 Experimental angular dependence of the $p$-polarised fluorescence intensity for a solution processed TPBi/OPV7 (10 wt\%) layer (solid circles) compared to simulated profiles with different anisotropy factors (solid line for $\theta_{\mathrm{v}}=0.2$; dash-dotted line for fully horizontal orientation, $\theta_{\mathrm{v}}=0$; dashed line for isotropic orientation, $\theta_{\mathrm{v}}=0.33$ ).

In Fig. 5 we show simulated intensity profiles for different orientational distributions of transition dipole moments. For fully randomly oriented transition dipole moments with an isotropic distribution function, the relative intensity at $57^{\circ}$ is 1.1. For the case where all transition dipoles are aligned perfectly in the plane of the film, the simulations predict a much lower relative intensity of the $p$-polarized fluorescence with a local maximum of 0.55 at $58^{\circ}$. Fig. 5 also shows the experimental data for the films containing $10 \mathrm{wt} \%$ OPV7. The data points lie in between the simulated traces for random and horizontal orientation, indicating a partial alignment of the transition dipole moments in the horizontal direction.

Using optical simulations we calculated the $p$-polarized intensity profile originating from the fluorescence of the organic layer assuming a distribution of transition dipole moments oriented along the vertical and horizontal directions with adjustable weight coefficients $\left(\theta_{\mathrm{v}}\right.$ and $\left.\theta_{\mathrm{h}}\right)$ to describe the prevalence of either of these orientations. For a fully random distribution of transition dipole moments, the coefficients for vertical and horizontal orientation are $\theta_{\mathrm{v}}=0.33$ and $\theta_{\mathrm{h}}=0.66$, respectively. Taking the experimental data for the TPBi/OPV7 (10 wt\%) and varying the coefficients in the simulations we find that the experimental data are best described by $\theta_{\mathrm{v}}=0.2$ and $\theta_{\mathrm{h}}=0.8$, i.e. a combination of $20 \%$ vertically and $80 \%$ horizontally aligned transition dipole moments. We note that although we consider the fit acceptable for the purpose of determining $\theta_{\mathrm{v}}$, it is not perfect. In the small-angle region the simulations predict a lower intensity than that found experimentally, and the peak in the high-angle range is at slightly higher angles in the simulations than in the experiment. Slight variations in the thickness of the films in the simulations did not affect these deviations.

The angular dependence of the $p$-polarised fluorescence profiles of the layers was analysed for all OPV $n$ compounds The comparison of the experimental and simulated angular dependence of the $p$-polarised fluorescence for the other OPV $n / \mathrm{TPBi}$ blends can be found in the ESI. $\dagger$ The fitting, obtained through the simulation software Setfos, is based on a chi-square minimization that takes all the measurement points into account between $0^{\circ}$ and $80^{\circ}$. A Levenberg-Marquardt algorithm is used to solve this non-linear problem. The simulated curves (see ESI $\dagger$ ) do not give a perfect fit to the data (which are consistently somewhat

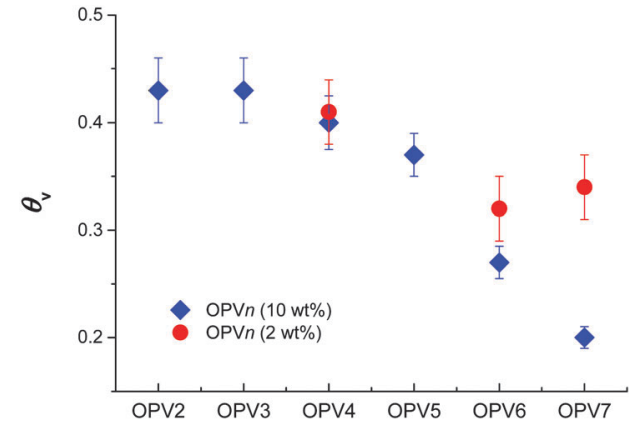

Fig. 6 Coefficient for vertical orientation $\left(\theta_{v}\right)$ of transition dipole moments in TPBi/OPVn host/guest layers obtained by fitting the experimental angular $p$-polarised fluorescence intensity to simulated profiles for partial horizontal $\left(\theta_{\mathrm{h}}\right)$ and vertical $\left(\theta_{\mathrm{v}}\right)$ orientation.

overestimated at angles below $20^{\circ}$ and between $50^{\circ}$ and $60^{\circ}$, and underestimated for complementary angles), but reproduce the salient features. The orientation coefficients $\theta_{\mathrm{v}}$ obtained in this way are shown in Fig. 6. In the layers containing the shortest emitters, with $n=2$ to 5 , there is a bias towards the vertical orientation of the fluorescent molecules $\left(\theta_{\mathrm{v}}>0.33\right)$. The physical basis for this preferred vertical orientation is presently not known. We cannot exclude that the observed $\theta_{\mathrm{v}}>0.33$ is a consequence of the density of photon modes favouring radiative decay from vertically aligned dipoles in these dielectric microcavity structures. ${ }^{27,28}$ Layers containing $10 \mathrm{wt} \%$ of OPV6 or OPV7 show a predominance of horizontally oriented emitting transition dipole moments $\left(\theta_{\mathrm{v}}<0.33\right)$.

In order to understand why the longest emitters show a preferential horizontal orientation of the emitting transition dipole moments, the photophysical properties and the morphology of the layers containing OPV6 or OPV7 were studied in more detail. The absorption spectra of OPV6 and OPV7 shown in Fig. 7 reveal a maximum in intensity around a wavelength of $430 \mathrm{~nm}$ for both oligomers. When dissolved in 2-MeTHF, OPV6 and OPV7 show maximum absorption at wavelengths of 466 and $473 \mathrm{~nm}$, respectively (Fig. S2, ESI $\dagger$ ). ${ }^{29}$ The absorption maximum of OPV6 and OPV7 in the film is thus strongly blue shifted even in comparison with the absorption maximum of OPV4 in the film. Furthermore the absorption spectra of the films containing $10 \mathrm{wt} \%$ of these longer oligomers also show a shift of the onset of absorption to longer wavelengths in comparison with the solution data as shown in Fig. 7. These spectroscopic features are characteristic of the formation of H-type aggregates for OPV6 and OPV7 in $10 \mathrm{wt} \%$ films with TPBi. $^{30}$ The presence of aggregates is further supported by fluorescence measurements. The spectra show a red shift of the emission band of the films in comparison to solution, which is consistent with H-type aggregation. ${ }^{30}$

Aggregation can be suppressed by lowering the concentration of the oligomers in the solution used to prepare the films. Fig. 8 shows the fluorescence spectra of TPBi/OPV6 and TPBi/OPV7 blends prepared from solutions with a reduced weight content of the oligomers ( $2 \mathrm{wt} \%$ instead of $10 \mathrm{wt} \%)$. For the films with a lower loading we find that the maximum in 


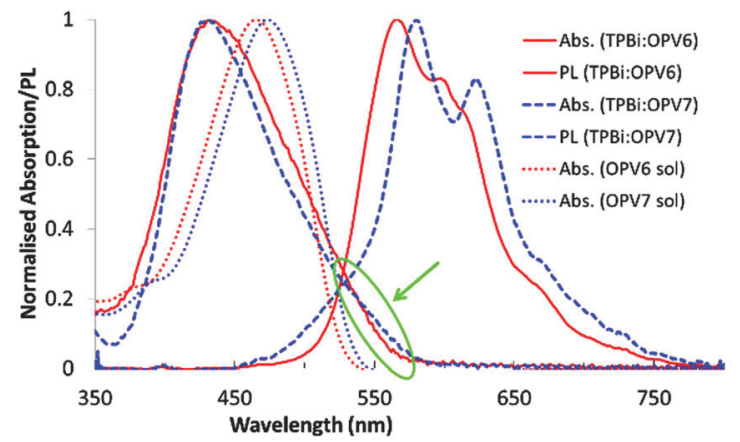

Fig. 7 Absorption and fluorescence spectra of TPBi/OPV6 10\% (solid lines) and TPBi/OPV7 (10 wt\%) (dashed lines) and of OPV6 and OPV7 in solution (dotted lines). The tail at high wavelengths in the absorption spectra of both the blends (circled area) indicates the aggregation of the films.

the fluorescence spectra occurs at shorter wavelengths in comparison with the films of higher loading. This indicates that the aggregation has been suppressed.

For films with low loading, containing only $2 \mathrm{wt} \%$ of OPV4, OPV6 and OPV7, the angular dependent fluorescence intensity profiles were determined (Fig. 9). For the shorter OPV4 oligomer there is practically no difference between the intensity profiles for high and low loading. Based on this result and the absorption spectra of the TPBi/OPV $n$ films (ESI $\dagger$ ) we conclude that for $n \leq 5$, the OPV $n$ molecules show no appreciable aggregation at $10 \mathrm{wt} \%$ concentration in TPBi. For the longest oligomers, OPV7, we find significantly higher relative intensity at an angle of $53^{\circ}$ for the film with low loading. This shows that the preference for the horizontal orientation of the OPV7 molecules in the layer is reduced at lower concentration. Analysis of the orientation factor using the simulation software gives $\theta_{\mathrm{v}}=0.34$ at $2 \mathrm{wt} \%$ loading which indicates a more isotropic orientation in comparison with the data for $10 \%$ loading $\left(\theta_{\mathrm{v}}=0.2\right)$. For OPV6 we find a similar reduction of the tendency towards horizontal orientation upon lowering the oligomer content. Hence, for non-aggregated OPV $n$ in TPBi the experiments (Fig. 6) show a small effect of the length of the oligomer on the preferential orientation of the transition dipole moment in the film.

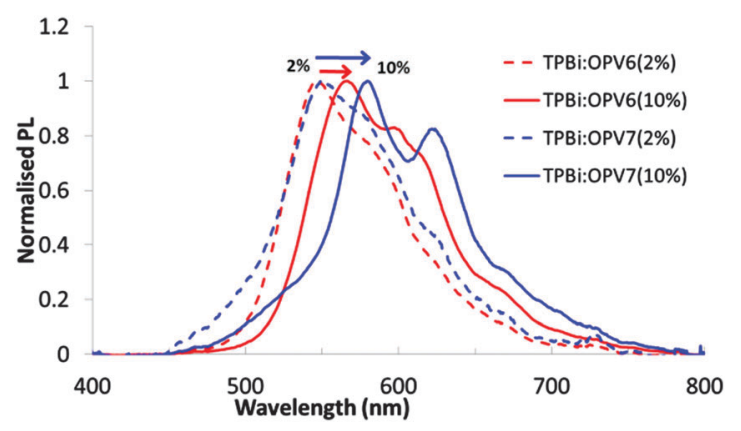

Fig. 8 Normalized fluorescence spectra of TPBi/OPV6 and TPBi/OPV7 host guest films with low loading ( $2 \mathrm{wt} \%$, dashed lines) and high loading (10 wt\%, solid lines).

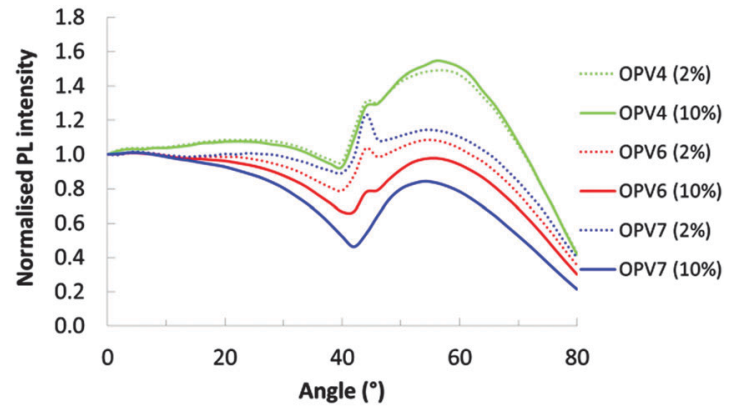

Fig. 9 Angular dependence of the $p$-polarised fluorescence intensity for a solution processed layer of TPBi/OPVn for low (2 wt\%) and high (10 wt\%) loading of the OPVn oligomer.

\section{Effect of aggregation on the orientation of the transition dipole moment}

To further understand the reason why the layers with the longest OPV $n$ molecules show a preferential horizontal orientation of transition dipole moments at high loading, we studied the morphology of the TPBi blend films containing OPV6 and OPV7 using AFM and TEM (Fig. 10). The TPBi layer with OPV6 at low concentration ( $2 \mathrm{wt} \%$ ) shows low surface roughness (the root mean square roughness $R_{\mathrm{q}}=0.49 \mathrm{~nm}$ for an area of $5 \mu \mathrm{m} \times$ $5 \mu \mathrm{m}$ ), and the low and small scale contrast in TEM indicates a homogeneous distribution of the guest inside the host material. In the blends with higher emitter content (10 wt\%), fibrillar structures several tens of $\mathrm{nm}$ in width and lengths typically in the range from 0.5 to a few $\mu \mathrm{m}$ can be observed by both AFM and TEM. Although the surface roughness is increased to $R_{\mathrm{q}}=3.41 \mathrm{~nm}$. These fibrillar structures are attributed to aggregates of the OPV6 that formed during the deposition of the layer. For films containing $2 \mathrm{wt} \%$ and $10 \mathrm{wt} \%$ OPV7 the surface roughness is $R_{\mathrm{q}}=0.57 \mathrm{~nm}$ and $R_{\mathrm{q}}=6.10 \mathrm{~nm}$, respectively. For the $10 \mathrm{wt} \%$ OPV7 film the fibrils have an increased length and width compared to films containing $10 \mathrm{wt} \%$ OPV6.

At higher concentration, the angular dependence of the $p$-polarised fluorescence of TPBi/OPV6 (10 wt $\%)$ and TPBi/OPV7 (10 wt\%) shows a predominance of the horizontal orientation

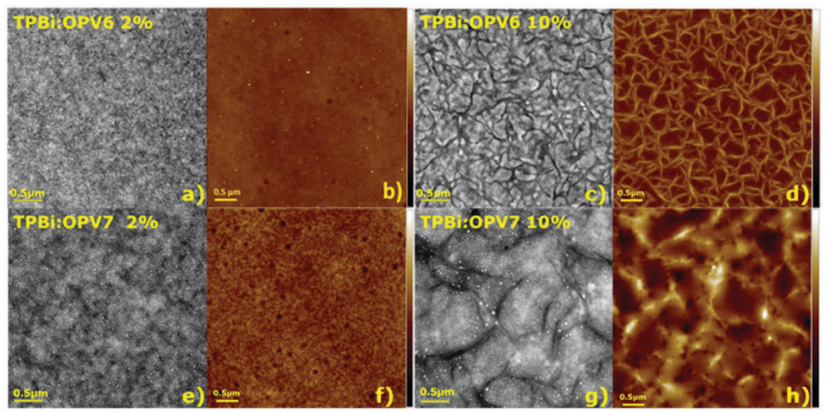

Fig. $10 \operatorname{TEM}(a, c, e$ and g) and AFM height (b, d, $f$ and $h$ ) images of $40 \mathrm{~nm}$ thick solution processed layers of ( $a$ and b) TPBi/OPV6 (2 wt\%), (c and d) TPBi/OPV6 (10 wt\%), (e and f) TPBi/OPV7 (2 wt\%) and ( $g$ and h) TPBi/OPV7 (10 wt\%). AFM images ( $b, d, f$ and $h$ ) show $5 \mu \mathrm{m} \times 5 \mu \mathrm{m}$ scans with the full colour scale corresponding to height differences of $0-10 \mathrm{~nm}$ in ( $b$ and $\mathrm{f}$ ) and $0-50 \mathrm{~nm}$ (in $\mathrm{d}$ and $\mathrm{h}$ ). 
of the emitting transition dipole moments in the thin film. The spectroscopic characteristics of the fluorescence from these films indicate that the photons emitted originate from aggregated guest molecules. Fibrillar aggregates of the guest molecules with a high aspect ratio can be observed directly in these films using microscopy. We tentatively assume that the predominantly in-plane orientation of the fibrils in the mixed film is related to the high aspect ratio. The correlation between preferred horizontal transition dipole moment orientation and aggregation is further substantiated by studying films with low loading and suggests that the predominance of horizontal orientation of the transition dipole moments may be brought about indirectly by the orientation of the aggregates in the films. A complete explanation of the horizontal alignment by a preferred orientation of the aggregates then requires that the transition dipole moments responsible for the emission are oriented predominantly along the long axis of the aggregate. Alternatively, the aggregates could also have a tape-like structure with the transition dipoles oriented in the plane of the tape and the tapes orienting preferentially flat on the substrate. Currently the internal structure of the aggregates of OPV6 and OPV7 is not known in detail. ${ }^{29}$ In these possible explanations, we have neglected any effects of photoselection of a preferred orientation of the emitting dipoles. In films of high loading, rapid Förster type energy transfer will occur, suppressing photoselection effects. For the films containing $10 \mathrm{wt} \%$ of OPV6 or OPV7, photoselection effects cannot be excluded, but are less likely to influence the results because of the high degree of in-plane orientation of the long aggregates. ${ }^{31}$

\section{Conclusions}

In summary, by studying the fluorescence properties of solution deposited thin films of a host/guest system and systematically varying the length of the guest molecules we find that the preferential horizontal orientation of the transition dipole moments for improved outcoupling efficiency in OLED systems may be realized by making use of the aggregation properties of the emitter molecules. We note that the fluorescence efficiency of the OPVn guest molecules is negatively affected by aggregation. As such these aggregated TPBi/OPVn films with horizontally aligned transition dipole moments are not particularly suited for OLED applications. Yet recent research on aggregation induced fluorescence and electroluminescence has shown that high luminescence quantum yields may be achieved in aggregating systems of chromophoric molecules which can result in the enhanced performance of OLEDs. ${ }^{32-35}$ In this respect it is of interest to combine chromophores that show aggregation induced emission and alignment of such aggregates to enhance the performance of solution-processed OLEDs.

\section{Acknowledgements}

The authors thank Horst Greiner (Philips) and Herman Schoo (Holst Centre) for useful discussions, and Marco van der Sluis (TU/e) for the technical support with the preparation of the measurement setup. The research of J. J. F. forms part of the research programme of the Dutch Polymer Institute (DPI), project \#734. This research has received funding from the Ministry of Education, Culture and Science (Gravity program 024.001.035).

\section{Notes and references}

1 K. Kato, T. Iwasaki and T. Tsujimura, J. Photopolym. Sci. Technol., 2015, 28, 335-340.

2 W. Brütting, J. Frischeisen, T. D. Schmidt, B. J. Scholz and C. Mayr, Phys. Status Solidi A, 2013, 210, 44-65.

3 P. Liehm, C. Murawski, M. Furno, B. Lüssem, K. Leo and M. C. Gather, Appl. Phys. Lett., 2012, 101, 253304.

4 M. Flämmich, J. Frischeisen, D. S. Setz, D. Michaelis, B. C. Krummacher, T. D. Schmidt, W. Brütting and N. Danz, Org. Electron., 2011, 12, 1663-1668.

5 J. Frischeisen, D. Yokoyama, A. Endo, C. Adachi and W. Brütting, Org. Electron., 2011, 12, 809-817.

6 C. Mayr, M. Taneda, C. Adachi and W. Brütting, Org. Electron., 2014, 15, 3031-3037.

7 D. Yokoyama, A. Sakaguchi, M. Suzuki and C. Adachi, Appl. Phys. Lett., 2009, 95, 243303.

8 D. Yokoyama, J. Mater. Chem., 2011, 21, 19187-19202.

9 D. Yokoyama, A. Sakaguchi, M. Suzuki and C. Adachi, Org. Electron., 2009, 10, 127-137.

10 D. Yokoyama, A. Sakaguchi, M. Suzuki and C. Adachi, Appl. Phys. Lett., 2008, 93, 173302.

11 D. Yokoyama, H. Sasabe, Y. Furukawa, C. Adachi and J. Kido, Adv. Funct. Mater., 2011, 21, 1375-1382.

12 A. Graf, P. Liehm, C. Murawsky, S. Hofmann, K. Leo and M. C. Gather, J. Mater. Chem. C, 2014, 2, 10298-10304.

13 M. J. Jurow, C. Mayr. T. D. Schmidt, T. Lampe, P. I. Djurovich, W. Brütting and M. E. Thompson, Nat. Mater., 2016, 15, 85-91.

14 D. McBranch, I. H. Campbell, D. L. Smith and J. P. Ferraris, Appl. Phys. Lett., 1975, 66, 1175-1177.

15 J. Sturm, S. Tasch, A. Niko, G. Leising, E. Toussaere, J. Zyss, T. C. Kowalczyk, K. D. Singer, U. Scherf and J. Huber, Thin Solid Films, 1997, 298, 138-142.

16 M. Tammer and A. P. Monkman, Adv. Mater., 2012, 24, 210-212.

17 C. M. Ramsdale and N. C. Greenham, Adv. Mater., 2012, 24, 212-215.

18 L. Zhao, T. Komino, M. Inoue, J.-H. Kim, J. C. Ribierre and C. Adachi, Appl. Phys. Lett., 2015, 106, 063301.

19 M. Shibata, Y. Sakai and D. Yokoyama, J. Mater. Chem. C, 2015, 3, 11187-11191.

20 Y. Sakai, M. Shibata and D. Yokoyama, Appl. Phys. Express, 2015, 8, 096601.

21 E. Peeters, R. A. J. Janssen, S. C. J. Meskers and E. W. Meijer, Polym. Prepr., 1999, 40, 519-520.

22 J. Frischeisen, D. Yokoyama, C. Adachi and W. Brütting, Appl. Phys. Lett., 2010, 96, 073302.

23 B. Ruhstaller, E. Knapp, B. Perucco, N. Reinke, D. Rezzonico and F. Müller, in Optoelectronic Devices and Properties, ed. O. Sergiyenko, InTech, Croatia, 2011, ch. 21, pp. 433-458. 
24 T. W. Hagler, K. Pakbaz and A. J. Heeger, Phys. Rev. B: Condens. Matter Mater. Phys., 1994, 49, 10968-10975.

25 M. Chandross, S. Mazumdar, M. Liess, P. A. Lane, Z. V. Vardeny, M. Hamaguchi and K. Yoshino, Phys. Rev. B: Condens. Matter Mater. Phys., 1997, 55, 1486-1496.

26 S. A. Mewes, J.-M. Mewes, A. Dreuw and F. Plasser, Phys. Chem. Chem. Phys., 2016, 18, 2548-2563.

27 G. Bjork, S. Machida, Y. Yamamoto and K. Igeta, Phys. Rev. A: At., Mol., Opt. Phys., 1991, 44, 669-681.

28 M. Furno, R. Meerheim, S. Hofmann, B. Lüssem and K. Leo, Phys. Rev. B: Condens. Matter Mater. Phys., 2012, 85, 115205.

29 E. Peeters, A. Marcos, S. C. J. Meskers and R. A. J. Janssen, J. Chem. Phys., 2000, 112, 9445-9454.
30 F. C. Spano, Annu. Rev. Phys. Chem., 2006, 57, 217-243.

31 S. C. J. Meskers, R. A. J. Janssen, J. E. M. Haverkort and J. H. Wolter, Chem. Phys., 2000, 260, 415-439.

32 Y. Hong, J. W. Y. Lam and B. Z. Tang, Chem. Soc. Rev., 2011, 40, 5361-5388.

33 C.-W. Lin and C.-T. Chen, in Aggregation-Induced Emission* Applications, ed. A. Qin and B. Z. Tang, John Wiley \& Sons, Ltd, Chichester UK, 2013, 1st edn, ch. 1, pp. 1-41.

34 H. Nie, B. Chen, C. Quan, J. Zhou, H. Qiu, R. Hu, S.-J. Su, A. Qin, Z. Zhao and B. Z. Tang, Chem. - Eur. J., 2015, 21, 8137-8147.

35 Y. Kubota, K. Kasatani, H. Takai, K. Funabikia and M. Matsui, Dalton Trans., 2015, 44, 3326-3341. 